\title{
The Impact of Ultra-widefield Retinal Imaging on Practice Efficiency
}

\author{
Paul E Tornambe \\ Retina Consultants, San Diego, California, US, and San Diego Retina Research Foundation, San Diego, California, US
}

DOI: https://doi.org/10.17925/USOR.2017.10.01.27

\begin{abstract}
$\mathrm{n}$ the current cost- and resource-constrained healthcare environment in the United States, characterized by declining government reimbursement and increased utilization scrutiny by managed care plans, providers are challenged to continue delivering quality care to more patients while also more effectively managing practice economics. Employing technology to improve practice efficiency is one of the most promising solutions to this dilemma. We have demonstrated that the integration of ultra-widefield (UWF) retinal imaging in our practice is cost-effective. It has allowed us to increase the number of patient encounters while simultaneously raising the quality of care, and increasing patient satisfaction.
\end{abstract}

\section{Keywords}

Ultra-widefield retinal imaging, UWF, practice efficiency, patient volume, cost-effectiveness, quality care, retinal periphery, patient satisfaction

Disclosure: Paul E Tornambe is a consultant to Optos.

Acknowledgements: Editorial assistance was provided by BioComm Network, Inc.

Compliance with Ethics: This study is based on data collected within the practice over a three year period as well as a review of the literature and did not involve any studies with human or animal subjects performed by the author.

Authorship:: The named author meets the International Committee of Medical Journal Editors (ICMJE) criteria for authorship of this manuscript, takes responsibility for the integrity of the work as a whole, and has given final approval to the version to be published.

open Access: This article is published under the Creative Commons Attribution Noncommercial License, which permits any noncommercial use, distribution, adaptation, and reproduction provided the original author(s) and source are given appropriate credit.

Received: February 6, 2017

Accepted: March 10, 2017

Citation: US Ophthalmic Review, 2017;10(1):27-30

Corresponding Author: Paul E Tornambe, 12630 Monte Vista Rd, Poway, CA 92064, US. E:tornambepe@aol.com

Support: The publication of this article was supported by Optos. The views and opinions expressed are those of the author and do not necessarily reflect those of Optos.
Ophthalmic medical practices in the United States are under unprecedented pressure to care for a rapidly growing population of patients, even as Medicare reimbursement levels continue to decline and payers are implementing payment programs based on cost and quality metrics. The Centers for Medicare and Medicaid Services (CMS) has continued to enact significant cuts to payment rates for a range of ophthalmic procedures, from glaucoma surgery to retinal detachment repair and various diagnostic imaging procedures. These are only the latest changes to the economics of care delivery to which ophthalmologists must rapidly adjust. More than ever, the financial viability of the ophthalmology practice depends on its ability to deliver efficient care - integrating cost, quality, outcomes, and patient satisfaction - within an ever-changing landscape of medical innovation, government regulation, payer intervention, and patient expectation.

Several years ago, we formally evaluated the relative efficiency of academic hospital center and small group practices by applying activity-based cost analysis to both settings. ${ }^{1}$ We found that the small group practice outperformed the academic medical center on nearly all markers of efficiency. In the latter setting, only four service lines - non-laser surgery (e.g., pneumatic retinopexy, pars plana vitrectomy, scleral buckling surgery), laser surgery (e.g., pan-retinal photocoagulation, retinal tear repair), non-optical coherence tomography (OCT) diagnostics (e.g., other forms of multi-modal imaging such as color, red free, autofluorescence, fluorescein angiography [FA], ultrasound), and injections - were profitable, with profit margins ranging from $62 \%$ for non-laser surgery to $1 \%$ for intravitreal injections. The largest negative profit contributions were generated by office visits and OCT imaging. The continued reduction of reimbursement rates for both diagnostic services and therapeutic injections in the intervening years has made conditions even more challenging for both types of practice and has increased the critical importance of evaluating and improving efficiency. Various models for measuring and comparing practicing efficiency are in development. For example, an efficiency index, calculated as a function of cost, number of patients receiving care, and the quality of care, has been proposed to measure the care delivery process for a given intervention within the ophthalmic practice. ${ }^{2}$ As such models are expanded to include outcome measures and are validated in larger inter-practice comparisons they may be useful in efforts to improve both the quality and efficiency of care.

Regardless of how efficiency is measured, it is increasingly clear that one key to the challenge of simultaneously improving practice efficiency and quality of care amid relentless financial pressure is the use of advanced technology - diagnostic and treatment systems that provide more useful clinical information, more rapidly, and with greater comfort or convenience for patients. An example along these lines is the positive impact on efficiency and quality of care in cataract and refractive practices produced by the integration of wavefront technology. The impact of this technology for digital refraction and optical path diagnostics was recently described, with the authors noting that the 
Figure 1: optomap of a retinal hole and horseshoe tear with a cuff of subretinal fluid found in the inferior temporal periphery

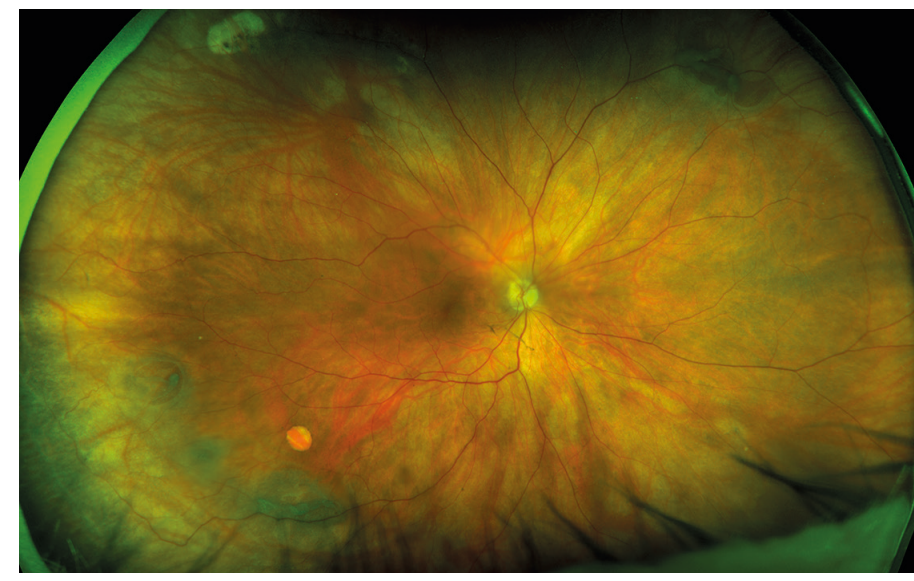

Image courtesy of William Keeling, MD.

Figure 2: optomap showing a temporal retinal detachment with a horseshoe tear in the far superior periphery

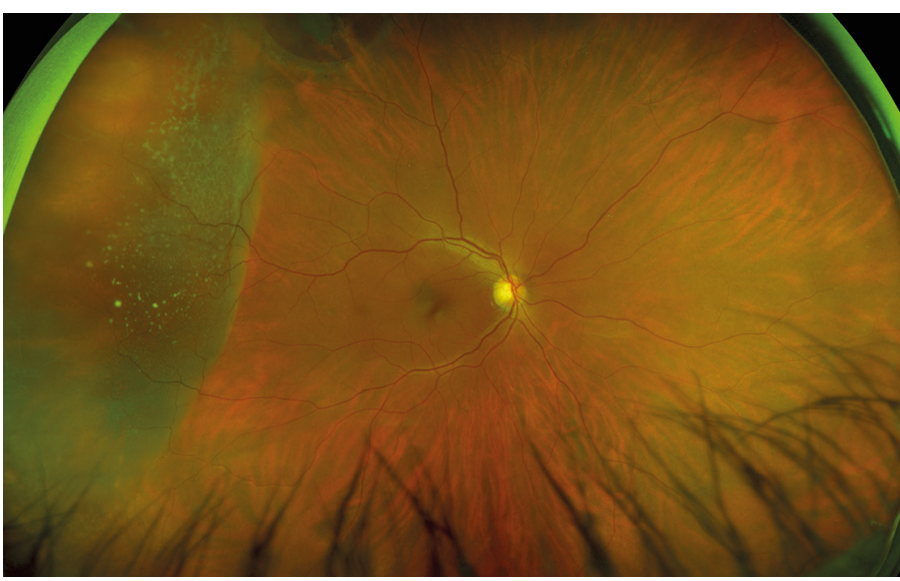

Figure 3: optomap demonstrating a choroidal melanoma nasal to the disc with peripapillary lipid exudation

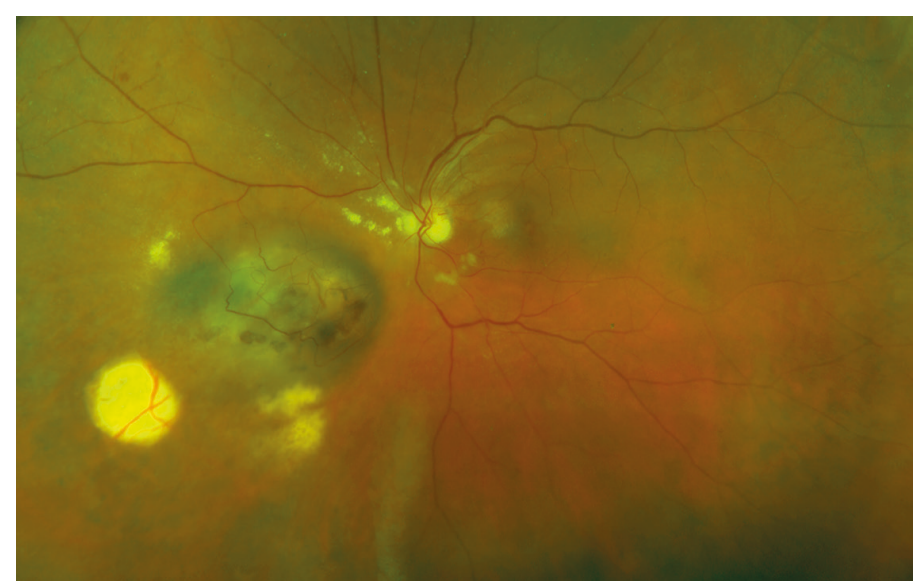

Image courtesy of Duke Eye Center. speed and comprehensiveness of total visual system assessment improved outcomes, patient satisfaction and cost-effectiveness. ${ }^{3}$ We have seen analogous benefits in our practice from the routine use of ultra-widefield (UWF ${ }^{\mathrm{TM}}$ ) retinal imaging using the Optos system. This paper will describe how the integration of UWF retinal imaging improved efficiency and quality of care, enhanced patient satisfaction, and allowed more patients to be examined during a fixed time interval in our practice. We show that the resulting increase in patient volume can improve cash flow irrespective of reimbursement rates or how payments for testing are bundled.

\section{Elements of efficiency for diagnostic imaging}

The unparalleled field of view (sometimes called "pan-retinal" imaging), rapid, patient-friendly image capture, high-resolution, and easy transmission of UWF optomap ${ }^{\circledR}$ (Optos, plc. Dunfermline, Scotland) imaging make it well suited to enhancing the efficiency and quality of ophthalmic care. The Optos system provides the widest field of view of any retinal imaging platform. ${ }^{4}$ The high-resolution digital image it produces is obtained in a single, non-contact, often non-mydriatic capture lasting about a second; the image is immediately available for evaluation by the clinician and review with the patient on a computer screen or tablet. Cross-registration of different imaging modalities or prior images is instantaneous, supporting comprehensive evaluation of retinal pathology or disease progression. The systems are DICOM compatible and utilize cloud-based transmission and storage to facilitate image sharing with other offices and practices, as well as archiving as part of the medical record.

A growing body of research has demonstrated the potential of UWF imaging to improve detection, documentation, and management of retinal pathology, particularly in zones of the periphery outside the field of view of conventional imaging. The potential of UWF imaging to improve the diagnosis and management of diabetic retinopathy (DR), one of our principle areas of clinical interest, is well documented. ${ }^{5}$ Multiple investigators have favorably compared UWF to conventional Early Treatment Diabetic Retinopathy Study (ETDRS) 7-fields, the standard DR assessment tool, observing that optomap images cover a much larger area of the retina, despite being acquired much more quickly and often without dilation. ${ }^{-8}$ Our own experience supports the conclusions of these and other studies which confirm that the use of UWF color imaging and UWF FA result in the detection of more pathology, including early signs of DR progression such as peripheral microaneurysms, neovascularization, vascular nonperfusion, and vascular leakage. These key indicators of disease severity might be missed or minimized by exams depending on standard field imaging. ${ }^{9-11}$ The greater accuracy and efficiency of UWF imaging has proven particularly useful in teleophthalmology. In one national ocular telehealth program focused on DR, nonmydriatic UWF imaging reduced the ungradable image rate by $81 \%$, nearly doubled the identification of DR, and identified peripheral lesions suggestive of more severe disease in nearly $10 \%$ of patients, compared to nonmydriatic multifield fundus photography. ${ }^{12}$

We have consistently seen similar benefits in our practice and have carefully evaluated the impact of UWF retinal imaging on the quality, costeffectiveness and revenue potential of the care we deliver, as well as its contribution to patient satisfaction.

\section{UWF improves the quality of care}

Fundamentally, quality in the context of the medical practice is doing the right thing, correctly, the first time. By this definition, each diagnostic 
procedure performed should provide the necessary support for the subsequent interventions. In examining the impact of UWF on quality, we should recognize that the optomap does not replace the retinal examination, particularly of the periphery, or scleral depression but it facilitates this examination by providing a pan-retinal overview allowing the clinician to focus attention on pathological findings of interest. Since the upper eye lid may limit visualization of the inferior retina, examination of the peripheral retinal is still necessary. The use of UWF also improves workflow efficiency by decreasing the technician time required for image acquisition and decreasing the time the patient must spend in the waiting room. Furthermore, as the extensive literature suggests, employing UWF first, on every patient, ensures that our team does not miss pathology, such as melanomas, retinal detachments, or DR, even on the busiest clinic day (see Figures 1-3). UWF imaging often reveals pathology that might be overlooked during the clinical exam, including signs of systemic disease, such as subtle vascular sheathing or rare microaneurysms, suggestive of diabetes, hypertension or carotid disease, in patients with no such diagnosis or symptoms. In fact, our experience reflects published studies that suggest UWF retinal imaging can be an outstanding screening tool - rapid and easy to perform, convenient for the patient, clinically useful, and readily shared. Finally, in situations with media changes, such as gas bubbles following retinal surgery, or in patients with small pupils or those who cannot tolerate a full exam, UWF provides the most effective way to examine the retina.

\section{UWF improves the cost-effectiveness of care}

When considering cost-effectiveness, clinicians must assess whether the revenue produced by a new technology is sufficient to cover the cost of its acquisition and whether a particular unit of care can be delivered in a profitable way for the practice. This means it must be acceptable to the payer and reimbursed at a revenue positive level. We have examined several potential impacts of UWF retinal imaging on revenue drivers in our practice, including its potential to increase the number of billable photographs and angiograms we take and the number of laser treatments we perform. We reviewed yearly patient encounters prior to and one and two years following the installation of our UWF retinal imaging system. With the use of UWF, the absolute number of photographs taken has increased; however, since not every image is considered medically-necessary by payer rules, the revenue contribution of this increase was relatively minor (for our analysis, we've assumed only a 50\% reimbursement rate). We also documented an increase in FA's and a small number of additional laser treatments, specifically in patients with non-proliferative DR in whom UWF had documented severe peripheral nonperfusion prior to the development of frank neovascularization. The volume change and revenue contributions of these three lines of service after the first year are summarized in Table 1.

By far, the most significant financial impact UWF has made in our practice is the increase in the number of patients we are able to see. This is largely the result of the reduction in the 'busy work' component of the office examination, such as noting cup-to-disc ratios, the presence of drusen, or retinal pigment epithelium and vascular changes. The use of UWF also facilitates more rapid determination of changes in peripheral lesions, including tumor growth, sheathing, or intraretinal microvascular abnormalities (IrMA). In the first year after we integrated UWF retinal imaging, we saw 220 more patients, an increase of $4.4 \%$ over the pre-UWF period, which was an average of 1.5 additional patient encounters per day.
Table 1: Change in procedure volume and revenue contribution in the first year following the adoption of UWF retinal imaging (comparing the period 9/2009 through 2/2010 to $9 / 2010$ through $8 / 2011$ )

\begin{tabular}{|l|l|l|l|}
\hline Procedure & $\begin{array}{l}\text { Fundus } \\
\text { photography }\end{array}$ & FA & $\begin{array}{l}\text { Diabetic } \\
\text { laser Tx }\end{array}$ \\
\hline Numerical change & +631 & +79 & +2 \\
\hline Percent change & $+70 \%$ & $+2.2 \%$ & (NS) \\
\hline Revenue contribution* & $\$ 24,552 * *$ & $\$ 18,500$ & (NS) \\
\hline
\end{tabular}

*Reimbursement rates at the time of analysis were $\$ 77.82$ for fundus photography and $\$ 233.93$ for FA. ${ }^{* *}$ Assumes $50 \%$ were allowed by third-party payer. $F A=$ fluorescein angiography; NS = not significant; TX = treatment.

This translated over $\$ 40,000$ in incremental revenue. In the second year of UWF use, patient volume increased by $7 \%$. The total incremental revenue generated by the integration of UWF was approximately $\$ 76,000$ per year or $\$ 6,333$ per month. As a result, the Optos UWF retinal imaging system we purchased paid for itself in just over a year. After 4 years of routine UWF retinal imaging, we have an average exam time of 11 minutes and are able to see 45 patients on a clinic day, with high patient satisfaction. We are now incorporating two 'followers,' one to a scribe position, the other to care for the immediate needs of post-injection patients, which should further decrease physician 'busy work' time by several minutes per patient, increasing patient capacity to 55 to 60 patients per day. Obviously, the type of visit encounter will affect the number of patient daily encounters. We give all injections at the time of the exam visit, and frequently give bilateral injections for patient convenience. Given that reimbursement rates have continued to decline (in our area, fundus photography is now reimbursed at \$71 and FA at \$92), the contribution of patient volume to the economic equation is even more significant. Of course, the financial benefit of integrating UWF will vary by practice but our experience is generally consistent with anecdotal reports we have heard from other retina offices.

It is worth noting that the combination of declining reimbursement levels and managed care plan payment adjustments based on utilization make the traditional practice of performing multiple diagnostic tests counterproductive from a revenue standpoint. By providing more information from a single test, data which has a direct impact on the quality of care, and by allowing more patients to be examined, UWF retinal imaging represents a new economic model for the ophthalmic practice, based on seeing more patients, not ordering more tests. Discovery and documentation of retinal pathology with an initial optomap also supports reimbursement of subsequent confirmatory tests like a UWF-guided peripheral exam with an indirect ophthalmoscope or OCT. The irrefutable documentation of the status of the retina at the time of the visit may also have beneficial medicolegal implications.

\section{UWF increases patient satisfaction}

Particularly when compared to conventional dilated fundus photography, UWF retinal imaging positively impacts the patient experience. The scanning laser ophthalmoscope (SLO) light is much more comfortable than the bright flash of traditional photography and the speed of image capture significantly reduces the amount of time patients spend in the office not actually receiving care. The reduction of physical exam time and related 'busy work' through the use of UWF retinal imaging also increases the proportion of the visit available for establishing a positive patient/doctor 
relationship, as well as patient counseling and education. Furthermore, the value of showing a patient what their actual retina looks like, rather than trying to explain using drawings or models, cannot be overstated. For example, patients who present with a retinal tear can be educated about flashes and floaters with a short video and then see the pathology on their own optomap. This increases their sense of urgency and their willingness to follow the treatment plan. Reviewing their UWF image helps them appreciate the benefits of early detection and understand why prompt treatment is critical to protect their vision.

We have also seen firsthand that improved patient satisfaction provides additional support for the flow of new patients, as happy patients tell their families and friends about their experience. The overall impact of the full integration of UWF retinal imaging in our practice is an increase of $12 \%$ in patient volume.

\section{Conclusion}

In order to thrive in the current healthcare environment, ophthalmic practices must attract and manage larger numbers of patients while simultaneously delivering high-quality, cost-effective care. To balance this equation - to maintain healthy revenue streams while providing quality care - ophthalmologists must utilize technology that will improve practice efficiency. The integration of UWF retinal imaging to produce more clinically-relevant information, more rapidly and in a more patientfriendly manner, can make important contributions to multiple aspects of the efficiency challenge. $\square$
1. Murray $T G$, Tornambe $P$, Dugel $P$, Tong $K B$, Evaluation of economic efficiencies in clinical retina practice: activity-based cost analysis and modeling to determine impacts of changes in patient management, Clin Ophthalmol, 2011;5:913-25.

2. Chen A, Kim EA, Aigner DJ, et al., Index to estimate the efficiency of an ophthalmic practice, JAMA Ophthalmol, 2015;133:924-9.

3. Matossian C, Noreika JC, Total visual system assessment integrating wavefront technology in refractive examinations, uS Ophthalmic Review, 2016:9:41-5.

4. Witmer MT, Parlitsis G, Patel S, Kiss S, Comparison of ultrawidefield fluorescein angiography with the Heidelberg Spectralis noncontact ultra-widefield module versus the Optos ${ }^{\oplus}$ Optomap ${ }^{\circledast}$, Clin Ophthalmol, 2013;7:389-94.

5. Kaines A, Oliver S, Reddy S, Schwartz SD, Ultrawide angle angiography for the detection and management of diabetic retinopathy, Int Ophthalmol Clin, 2009:49:53-9.

6. Kernt M, Hadi I, Pinter F, et al., Assessment of diabetic retinopathy using nonmydriatic ultra-widefield scanning laser ophthalmoscopy (optomap) compared with ETDRS 7-field stereo photography, Diabetes Care, 2012;35:2459-63.

7. Kernt M, Pinter F, Hadi I, et al., Diabetic retinopathy: comparison of the diagnostic features of ultra-widefield scanning laser ophthalmoscopy Optomap with ETDRS 7-field fundus photography [article in German], Ophthalmologe, 2011;108:117-23.

8. Silva PS, Cavallerano JD, Sun JK, et al., Nonmydriatic ultrawide fiel retinal imaging compared with dilated standard 7-field $35-\mathrm{mm}$ photography and retinal specialist examination for evaluation of diabetic retinopathy, Am J Ophthalmol, 2012;154:549-59.
9. Wessel MM Aaker GD, Parlitsis $G$ et al Ultra-wide-field angiography improves the detection and classification of diabetic retinopathy, Retina, 2012;32:785-91.

10. Kong M, Lee MY, Ham DI, Ultrawide-field fluorescein angiography for evaluation of diabetic retinopathy, Korean I Ophthalmol, 2012;26:428-31.

11. Silva PS, Cavallerano JD, Sun JK, et al., Peripheral lesions identified by mydriatic ultrawide field imaging: distribution and potential impact on diabetic retinopathy severity, Ophthalmology, 2013;120:2587-95

12. Silva PS, Horton MB, Clary D, et al., Identification of diabetic retinopathy and ungradable image rate with ultrawide field imaging in a national teleophthalmology program, Ophthalmology, 2016;123:1-8 